\title{
Laparoscopic repair of giant paraesophageal hernia
}

Evan T. Alicuben, MD, James D. Luketich, MD, and Ryan M. Levy, MD, Pittsburgh, Pa

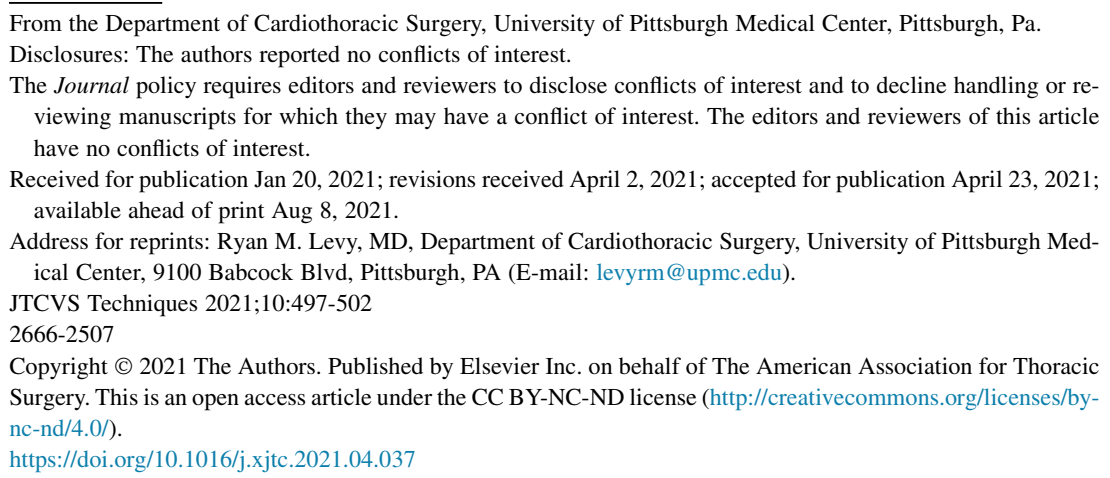

Video clip is available online.

Feature Editor's Introduction-Secondary to its intricacies and complexities, "benign esophageal surgery" is often considered its own surgical field, composed primarily of antireflux surgery, esophageal motility surgery, and surgery of the esophageal hiatus. Giant paraesophageal hernias are hiatal hernias in which greater than one third of the stomach is intrathoracic and their repair is arguably one of the most complex "benign esophageal" procedures. They are large (by definition), can contain multiple abdominal viscera, and may necessitate additional esophageal lengthening procedures. This is an operation In which technique is directly related to long-term outcomes, and they are prone to recurrence. In this Video Atlas Article, it is fitting that the laparoscopic technique of giant paraesophageal hernia repair is crystalized by pioneers in thoracic surgery. Thoracic surgeons are unduly qualified in open, minimally invasive, and robotic esophageal surgery, and are in fact, the surgeons who remove and replace the esophagus when required. It is anticipated that this VAA will be studied by trainees and early career surgeons and will provide pearls to even the most experienced esophageal surgeons.

\section{Bryan M. Burt, MD}

Giant paraesophageal hernias are defined by greater than $33 \%$ of the stomach herniating through the diaphragmatic esophageal hiatus into the mediastinum. These include type 3 (herniated gastroesophageal junction and stomach)

\section{METHODS}

\section{RESULTS}

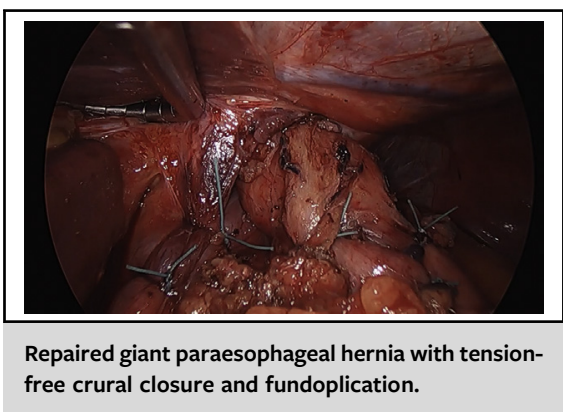

\section{CENTRAL MESSAGE \\ Components of the laparoscopic repair of the giant paraesopha- geal hernia strive to restore normal anatomy and achieve a tension free crural repair.}

See Commentaries on pages 503,505 , and 507

and type 4 hiatal hernias (herniated gastroesophageal junction, stomach and other organs, or omentum). Despite technical advances in minimally invasive techniques, recurrence rates following operative repair remain high in many series. We first described our initial laparoscopic series in $2000 .{ }^{1}$ Since that initial publication, we have refined and evolved our laparoscopic technique. Strict adherence to surgical principles of repair with carefully selected use of adjunctive techniques is key to achieving a low recurrence rate. These principles include minimizing tension, obtaining adequate length of intra-abdominal esophagus, preserving crural peritoneal lining, and fat pad/vagal nerve mobilization. Herein, we describe our technique in 10 steps.

The technique of laparoscopic repair of giant paraesophageal hernia repair is described in the following 10 steps, each with an accompanying video. Dissection is performed with the Harmonic scalpel and suturing is accomplished with the Endo Stitch device (Covidien, Minneapolis, Minn). This study received approval from our institutional review board (Evaluation of Outcomes Following Surgical Procedures for Benign Esophageal Disease, number: STUDY20010200) as part of our evaluation of outcomes following surgical procedures for benign esophageal diseases. Participants provided consent for use of their data collected.

The patient is placed in the supine position with arms extended at a neutral position. We ensure the patient is at 
the far right side of the table to facilitate use of a Lapro-Flex articulating liver retractor (Mediflex, Islandia, NY). A foot board with padding is placed to allow for steep reverse Trendelenburg. The surgeon stands on the patient's right with the first assistant on the left.

\section{Port Placement (Video 1)}

Most cases are performed with 5 ports. The upper abdomen is divided into thirds from the xiphoid to umbilicus. An open cutdown is performed in the right paramedian line around the upper third line, which equates to approximately 8 to $10 \mathrm{~cm}$ from the xiphoid. Through this incision, a Hasson port is placed and carbon dioxide insufflation is introduced. The patient is gradually placed into reverse Trendelenburg while insufflating to a maximum of 12 to $15 \mathrm{~mm} \mathrm{Hg}$.

The following additional ports are placed under direct visualization:

- a 10-mm port in the left paramedian line at the same level or slightly lower to the first Hasson port. The camera is placed through this port;

- a 5-mm port one hand's breath lateral to the camera port just below the costal margin for the assistant;

- a 5-mm port one hand's breath lateral to the Hasson port just below the costal margin for the surgeon's left hand; and

- a 5-mm port at the far lateral right abdominal wall just below the costal margin for the liver retractor.

- We use an additional 5-mm port in the periumbilical area in some cases for additional stomach retraction.

\section{Hernia Sac Reduction and Entry into the Mediastinal Plane (Video 2)}

The stomach and any other abdominal contents are carefully reduced into the abdomen. In many cases, the stomach can be chronically incarcerated, but typically some of it is reducible, allowing creation of a working space within the sac. This is the most common scenario in elective cases.

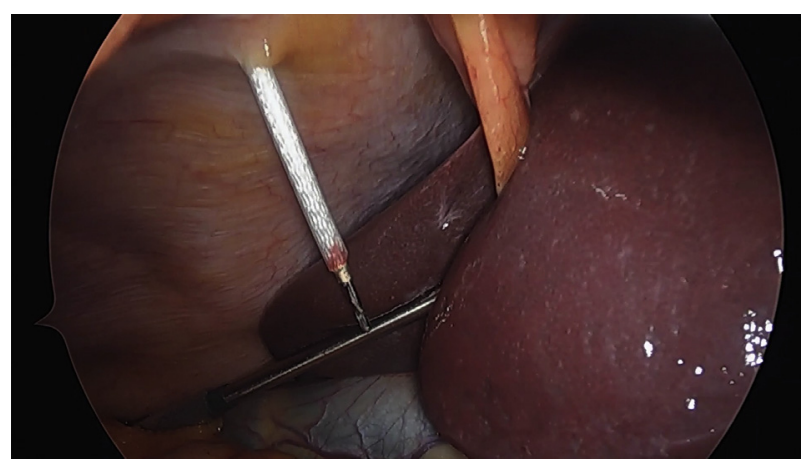

VIDEO 1. Video available at: https://www.jtcvs.org/article/S26662507(21)00514-9/fulltext.

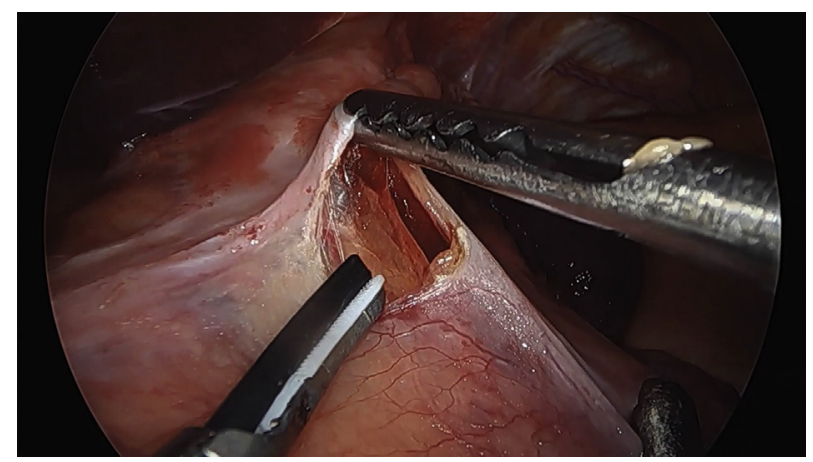

VIDEO 2. Video available at: https://www.jtcvs.org/article/S26662507(21)00514-9/fulltext.

If a type 4 hiatal hernia is present, we typically reduce herniated colon or omentum as the initial step. Grasping epiploic appendages for retraction will help to avoid injury to the colon. Dividing adhesions along the left crus may aid in being able to reduce a chronically incarcerated structure. The most proximal portion of the sac is grasped by both surgeon and assistant and everted until the transition from sac to normal peritoneum is visualized. Using the second assistant port to maintain downward traction on the stomach while sac eversion is being performed may be helpful. However, care should be taken to not use excess tension, as stomach tears may occur. An energy device is used to incise the hernia sac at the line of the peritoneal reflection entering the mediastinum anteriorly. Identification of the areolar tissue plane is critical but can be challenging. Working within this space translates to a relatively avascular plane maximizing visualization and avoiding injury to the yet to be identified vagus nerves.

\section{Mediastinal Mobilization (Video 3)}

Our next goal is complete $360^{\circ}$ esophageal mobilization. Dissection is performed anteriorly first working to develop the plane along the posterior pericardial surface. We then work to reduce the sac further and divide attachments to the left pleura. The anterior vagus nerve should be identified during these steps. This dissection proceeds in a posterior

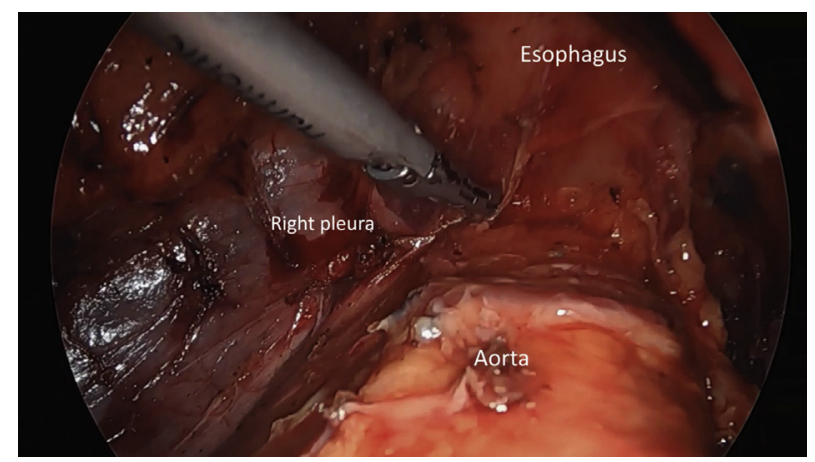

VIDEO 3. Video available at: https://www.jtcvs.org/article/S26662507(21)00514-9/fulltext. 
direction until the aorta is visualized. Maximizing the posterior dissection from the left side will allow for carbon dioxide insufflation to aid in the right side mobilization. We recommend dividing attachments with an energy source to avoid bleeding. In our experience, this can be done closely to the esophagus and stomach without causing thermal injury. Entry into the pleural space is avoided if possible, as this can impair visualization due to billowing pleura and possibly result in hemodynamic alterations. Both vagal nerves should be identified and preserved. Working outside of the esophageal fat pad as well as the hernia sac is critical for vagal nerve preservation. In addition, only areolar tissue planes should be separated and divided. In general, any adhesive bands that appear to run in a parallel direction to the esophagus should create pause in the surgeon's mind as a possible vagal nerve. Dissection is carried superiorly to above the level of the inferior pulmonary veins. It is critically important to not abandon the mediastinal dissection plane too early.

\section{Dissection of Lesser Curve and Right Crus (Video 4)}

Once mediastinal mobilization has been maximized, the surgeon returns to the abdominal phase of the procedure. The gastrohepatic ligament is divided at its avascular portion entering the lesser sac. Division of the ligament proceeds in a superior direction towards the right crus. As the right crus is approached, extreme care should be taken to identify a plane between the hernia sac and peritoneal lining to avoid denuding the crural pillar. Exposed muscle fibers can result in a challenging crural reapproximation and predispose to hernia recurrence. Dissection should continue so as to meet the plane of dissection from the initial mediastinal work. The remainder of the hernia sac attachments to the right crus and right pleura should be mobilized off and everted from their mediastinal attachments. Complete mobilization off of the aortic plane is also accomplished during this phase.

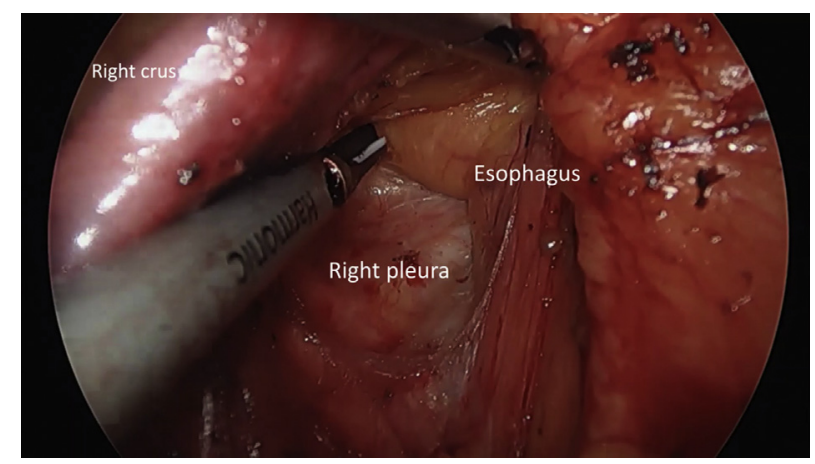

VIDEO 4. Video available at: https://www.jtcvs.org/article/S26662507(21)00514-9/fulltext.

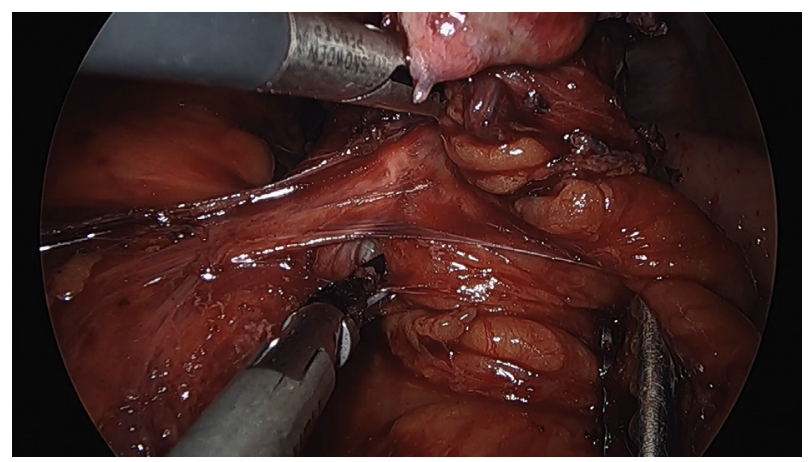

VIDEO 5. Video available at: https://www.jtcvs.org/article/S26662507(21)00514-9/fulltext.

\section{Dissection of Greater Curve and Left Crus, Division of Short Gastric Vessels (Video 5)}

The omentum is retracted laterally to identify the terminal branches of the gastroepiploic arcade differentiating them from the short gastric vessels. The vessels are then divided with an energy device with liberal use of clips for hemostasis. Care should be taken to divide these vessels close to the stomach and avoid injury to the spleen. As the left crus is approached, equal extreme care should be taken to preserve the overlying peritoneal lining. Mobilization of the thickened hernia sac attachments between the spleen and the left crus is important so as to result in a tension-free repair.

\section{Assessment of Esophageal Length (Video 6)}

Critical to achieving a tension-free repair is evaluation for 2.5 to $3 \mathrm{~cm}$ of intra-abdominal esophageal length. Given the possibility of tubularized cardia appearing as esophageal length, we routinely mobilize the esophageal fat pad to visualize the true gastroesophageal junction and angle of His. This also allows for mobilization of the anterior and posterior vagus nerves off the esophagus, thus permitting the fundoplication to sit inside of the fat pad and nerves. This can be done safely by staying in a plane close to the esophagus and gastric cardia, dividing only clear structures that course in a direction perpendicular to the esophagus.

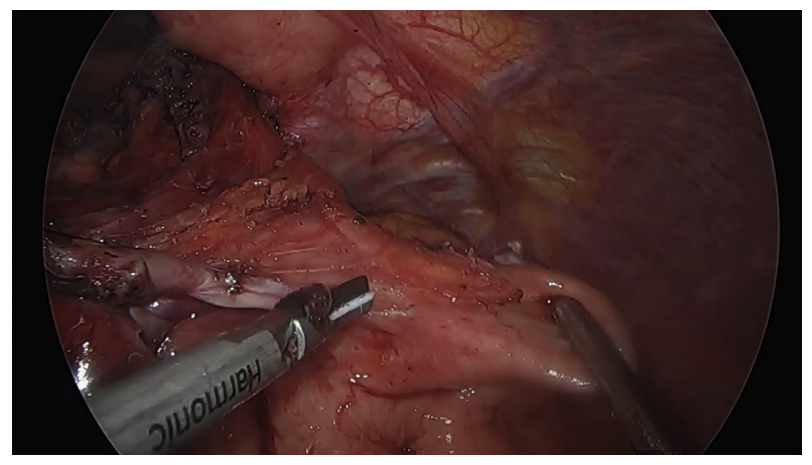

VIDEO 6. Video available at: https://www.jtcvs.org/article/S26662507(21)00514-9/fulltext. 


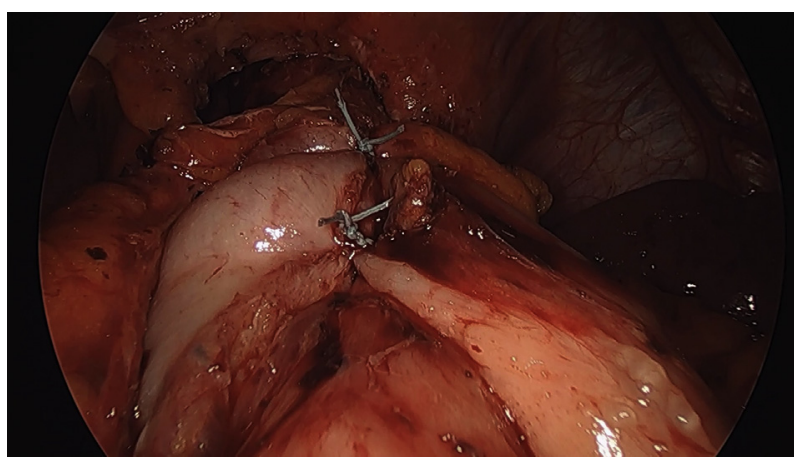

VIDEO 7. Video available at: https://www.jtcvs.org/article/S26662507(21)00514-9/fulltext.

Any band coursing in a parallel direction may be the vagus nerve and should not be divided until the nerve has been definitively identified. We believe that sewing the wrap to the peritoneal lined fat pad prevents the formation of scar tissue in the area to prevent wrap slippage. Thus, fat pad and nerve mobilization permit sewing directly to the esophagus. Moreover, particularly in overweight and obese patients, the fat pad can be quite bulky, so having this inside the wrap can be an impediment to proper placement. Also, if a Collis gastroplasty is performed, not mobilizing the anterior vagus nerve puts it at risk for injury during stapling. We routinely perform an upper endoscopy to correlate endoscopic and laparoscopic findings and measurements.

\section{Fundoplication (Video 7)}

If adequate intra-abdominal esophageal length $(\geq 2.5 \mathrm{~cm})$ has been established, a fundoplication is performed. A 54Fr bougie is passed under direct visualization, ensuring that the widest portion sits at the gastroesophageal junction. The surgeon passes a retroesophageal grasper inside of the posterior vagus nerve to which the fundus is grasped and brought through a window between the vagus and esophagus. A shoe-shine maneuver is performed to confirm the anterior stomach surface is still oriented anteriorly directly against the posterior esophageal surface. The wrap is then created with 2-0 Surgidac sutures.

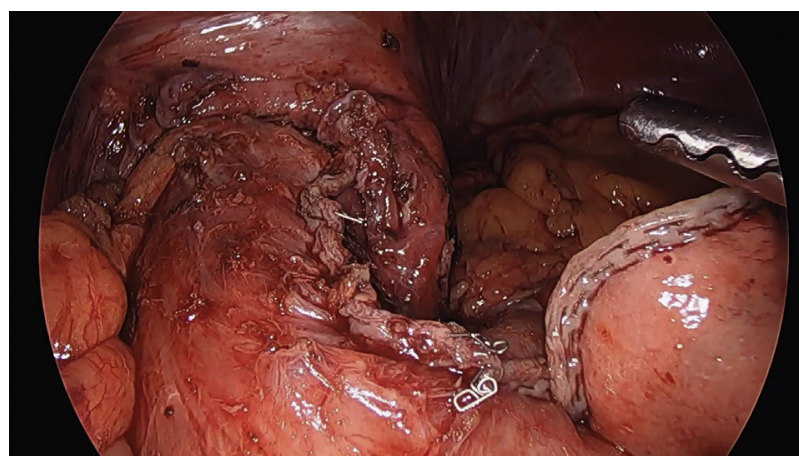

VIDEO 8. Video available at: https://www.jtcvs.org/article/S26662507(21)00514-9/fulltext.

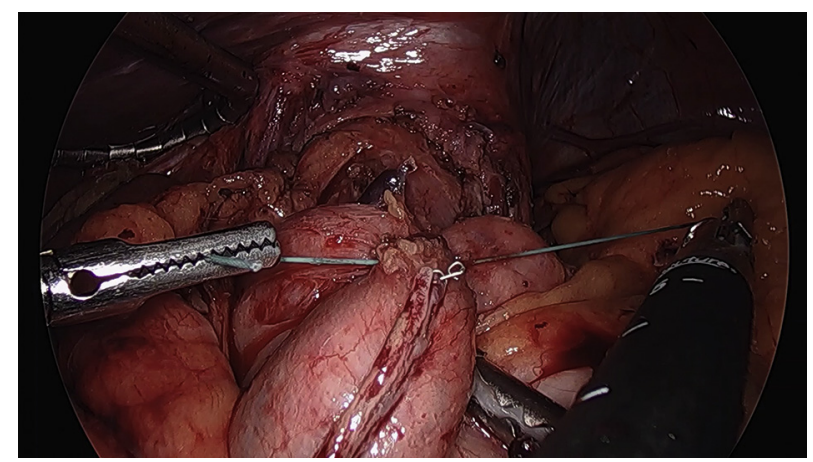

VIDEO 9. Video available at: https://www.jtcvs.org/article/S26662507(21)00514-9/fulltext.

\section{Collis Gastroplasty (Video 8)}

If the esophagus is foreshortened, a wedge type Collis gastroplasty is performed. A 54-Fr bougie is passed into the stomach ensuring that it sits along the lesser curve. Measurements are performed to determine the amount of stomach to be resected to create the neoesophagus. Our goal is to create $3 \mathrm{~cm}$ of intra-abdominal neoesophagus (Collis segment). This will allow the fundoplication to sit 2.5 to $3 \mathrm{~cm}$ below the diaphragm. It is important to not create more length than is necessary as resecting too much fundus will result in an overly tight fundoplication. A gastroplasty is performed by firing stapler loads perpendicular to the bougie until the bougie is pushed away by the stapler when closed. Parallel stapler loads are then fired along the bougie creating the neoesophagus segment. Subsequently, the neofundic tip can then be passed through the retroesophageal window for creation of the fundoplication (Video 9).

\section{Reapproximation of Crura (Video 10)}

The bougie is removed and tension-free crural reapproximation is then performed. While it is our normal practice to perform this step after fundoplication, it is also possible to close the crura before fundoplication. In general, we do not have issues with visualizing the crura after fundoplication.

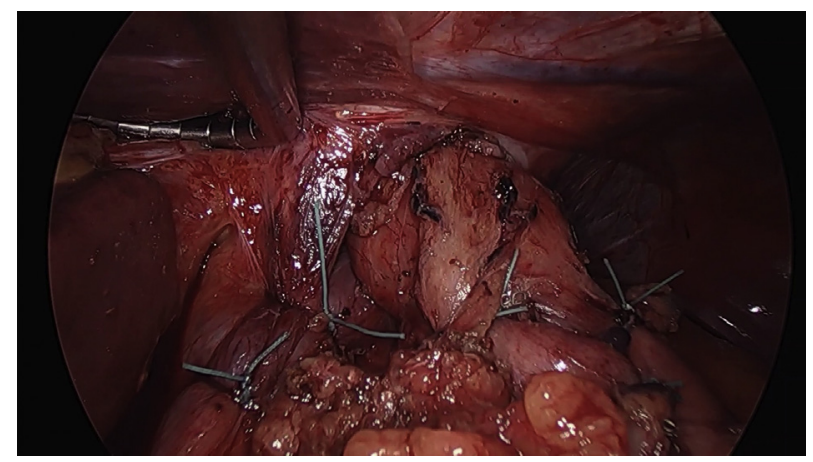

VIDEO 10. Video available at: https://www.jtcvs.org/article/S2666 2507(21)00514-9/fulltext. 
It is important to not grasp or put traction on the fundoplication itself. Instead, the assistant uses a closed grasper to elevate the fundoplication, esophagus and stomach to expose the crura. Preservation of the crural peritoneal lining is critical to achieving optimal closure. Generally, we place 2 posterior 0 Surgidac sutures and then assess the remaining hiatal opening. Anterior or lateral sutures are placed to restore a natural lie to the esophagus, avoiding a posterior ridge effect which can be seen with posterior only closure. In patients with attenuated crura, tension free closure can be difficult. To facilitate this, we sometimes deliberately induce a carbon dioxide pneumothorax in the left pleural space by placement of a 5-mm port into the left chest under direct visualization. Creation of a floppy diaphragm aids in placement of sutures to achieve a tension free repair. This technique can be particularly helpful in the redo setting with fibrotic crura that are not very mobile.

\section{Gastropexy (Video 11)}

For patients with giant paraesophageal hernias and primarily obstructive symptoms, we no longer routinely perform an anti-reflux procedure. Rather, in our current paradigm, patients with exclusively obstructive symptomatology (ie, postprandial pain or emesis, dysphagia, dyspnea, anemia, early satiety) undergo a gastropexy. It should be emphasized that for gastropexy, we still perform complete mediastinal mobilization and hernia sac reduction. Once these steps are completed, sutures are placed to recreate an angle of His. A series of horizontal mattress sutures are placed from the line of the short gastric vessels to an everted edge of the left hemidiaphragm that lies above the spleen. This line of sutures strives to recreate a normal anatomic lie of the stomach.

\section{DISCUSSION}

Operative repair of giant paraesophageal hernias has evolved to a minimally invasive technique. The safety and efficacy of this approach has been established in numerous studies. ${ }^{2}$ Nevertheless, recurrence rates have remained high

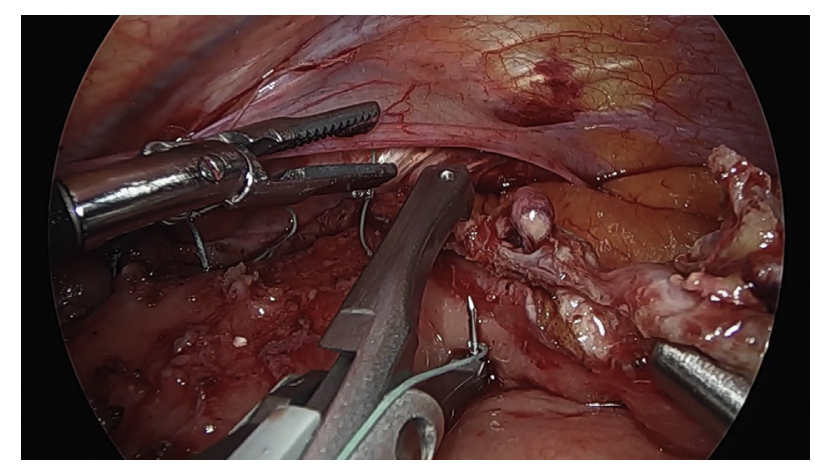

VIDEO 11. Video available at: https://www.jtcvs.org/article/S26662507(21)00514-9/fulltext. prompting continued interest in refining surgical techniques. Our approach has evolved to include a focus on aggressive mediastinal mobilization, accurate assessment of esophageal length through fat pad mobilization and preservation of the crural lining. ${ }^{3,4}$ Adherence to these principles was associated with a low recurrence rate and a low reoperation rate. ${ }^{2}$

The use of adjunctive techniques including collis gastroplasty and mesh reinforcement remains controversial. In patients with shortened esophagus, collis gastroplasty provides a means to minimize axial tension on the repair. Determination of need for this adjunct is only made after full mobilization of the esophageal fat pad for direct visualization of the true gastroesophageal junction. In our series of 662 giant paraesophageal hernia repairs published in 2010 , the rate of collis gastroplasty decreased over time but was 53\% in the group who had undergone more recent repair. Anecdotally, our present rate of collis gastroplasty is now lower than what we published 10 years ago. This is likely due to improvements in operative technique and experience gained over time.

Mesh reinforcement of hiatal closure was first shown to be effective in trials using synthetic material. ${ }^{5}$ Concerns over esophageal erosion prompted investigation into use of biologically compatible materials. ${ }^{6}$ While early results showed promising improvement in recurrence, this benefit did not persist in long-term follow-up. ${ }^{7,8}$ Thus, many have questioned the role of mesh. In our experience, we have found that mesh cruraplasty was not needed in most cases. Keys to avoiding the need for mesh include preservation of peritoneal integrity on the crural pillars and mobilization of all attachments to the left hemi-diaphragm. Following these principles, our last publication reported the need for mesh in only $13 \%$ of cases (typically needed when the integrity of the crura themselves were significantly threatened, mostly in reoperative cases). As we have improved our operative technique over time, our current rate of mesh use is considerably lower at present time.

Our paradigm for management of giant paraesophageal hernia continues to evolve with the incorporation of gastropexy instead of fundoplication for patients with primarily obstructive symptoms. It is critically important to note that all other components of the operation remain identical, including full mediastinal mobilization and primary crural reapproximation. Consideration for this approach may be warranted if the appropriate symptom complex is present. Moreover, this "complete mobilization gastropexy" may be a preferable option for the elderly giant paraesophageal hernia patients and patients wanting to avoid fundoplication.

\section{CONCLUSIONS}

Successful laparoscopic repair of giant paraesophageal hernia focuses on operative steps that restore normal anatomy and allow for a crural repair that is tension 
free. Following these principles minimizes risk for recurrence.

\section{References}

1. Luketich JD, Raja S, Fernando HC, Campbell W, Christie NA, Buenaventura PO, et al. Laparoscopic repair of giant paraesophageal hernia: 100 consecutive cases. Ann Surg. 2000;232:608-18.

2. Luketich JD, Nason KS, Christie NA, Pennathur A, Jobe BA, Landreneau RJ, et al. Outcomes after a decade of laparoscopic giant paraesophageal hernia repair. $J$ Thorac Cardiovasc Surg. 2010;139:395-404. 404.e1.

3. Chan EG, Sarkaria IS, Luketich JD, Levy R. Laparoscopic approach to paraesophageal hernia repair. Thorac Surg Clin. 2019;29:395-403.

4. Nason KS, Luketich JD, Witteman BP, Levy RM. The laparoscopic approach to paraesophageal hernia repair. J Gastrointest Surg. 2012;16:417-26.
5. Granderath FA, Schweiger UM, Kamolz T, Pasiut M, Haas CF, Pointner R Laparoscopic antireflux surgery with routine mesh-hiatoplasty in the treatment of gastroesophageal reflux disease. J Gastrointest Surg. 2002;6: 347-53.

6. Stadlhuber RJ, Sherif AE, Mittal SK, Fitzgibbons RJ Jr, Brunt LM, Hunter JG, et al. Mesh complications after prosthetic reinforcement of hiatal closure: a 28case series. Surg Endosc. 2009;23:1219-26.

7. Oelschlager BK, Pellegrini CA, Hunter J, Soper N, Brunt M, Sheppard B et al. Biologic prosthesis reduces recurrence after laparoscopic paraesophageal hernia repair: a multicenter, prospective, randomized trial. Ann Surg. 2006; 244:481-90.

8. Oelschlager BK, Pellegrini CA, Hunter JG, Brunt ML, Soper NJ, Sheppard BC, et al. Biologic prosthesis to prevent recurrence after laparoscopic paraesophageal hernia repair: long-term follow-up from a multicenter, prospective, randomized trial. J Am Coll Surg. 2011;213:461-8. 